$\square$ Math-Net.Ru

Общероссийский математический портал 
Р. Яйте, Индивидуальная эргодическая теорема для сжатий в пространствах $\mathbb{L}_{p}(\mathcal{H})$, Функи. анализ и его прил., 2006, том 40, выпуск 2, 90-94

DOI: https://doi.org/10.4213/faa13

Использование Общероссийского математического портала Math-Net.Ru 
подразумевает, что вы прочитали и согласны с пользовательским соглашением

http://www.mathnet.ru/rus/agreement

Параметры загрузки:

IP : 3.85 .7 .115

26 апреля 2023 г., 18:34:07 


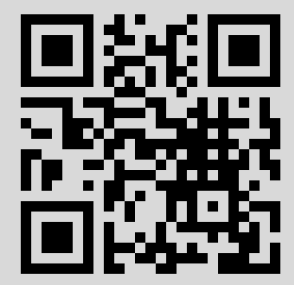


УДК 517.98

\section{Индивидуальная эргодическая теорема для сжатий в пространствах $\mathbb{L}_{p}(\mathscr{H})$}

(c) 2006. Р. ЯйтЕ

1. Введение и основной результат. Пусть $\mathscr{H}$ - комплексное сепарабельное гильбертово пространство. Обозначим через $\mathbb{L}_{p}(Z, \mathscr{F}, \mu ; \mathscr{H})$ пространство $\mathscr{H}$-значных измеримых функций $\xi$ на конечном пространстве с мерой $(Z, \mathscr{F}, \mu)$, таких, что норма

$$
\|\xi\|_{p}=\left(\int_{Z}\|\xi(z)\|_{\mathscr{H}}^{p} \mu(d z)\right)^{1 / p}
$$


конечна. В предположении, что $1<p<\infty$ и $p \neq 2$, мы доказываем результат о поточечной сходимости для сжатий в пространстве $\mathbb{L}_{p}(Z, \mathscr{F}, \mu ; \mathscr{H})$, тесно связанный со знаменитой эргодической теоремой Акоглу для положительных сжатий в классических $\mathbb{L}_{p}$-пространствах [1].

Начнем с необходимых обозначений и определений. Пусть $(Z, \mu)$ - пространство с чисто атомической мерой, имеющей конечное число атомов $m_{1}, \ldots, m_{n}$, т. е. $Z=\{1, \ldots, n\}$ и $\mu\{j\}=m_{j}$. Обозначим через $\mathbb{L}_{p}\left(m_{1}, \ldots, m_{n} ; \mathscr{H}\right)$ соответствующее $\mathbb{L}_{p}$-пространство. Линейный оператор $T$ в пространстве $\mathbb{L}_{p}\left(m_{1}, \ldots\right.$, $\left.m_{n} ; \mathscr{H}\right)$ задается матрицей $T=\left(T_{i j}\right)_{i, j=1, \ldots, n}$ линейных операторов в $\mathscr{H}$, т. е. для $f=\left(f_{1}, \ldots, f_{n}\right), f_{i} \in \mathscr{H}$,

$$
(T f)_{j}=\sum_{i=1}^{n} T_{i j} f_{i}, \quad j=1, \ldots, n .
$$

ОПРЕДЕЛЕНИЕ. Будем говорить, что оператор $T=\left(T_{i j}\right)$ в пространстве $\mathbb{L}_{p}\left(m_{1}, \ldots, m_{n} ; \mathscr{H}\right)$ мажсорируется сжатием, если

$$
\left\|T_{i j}\right\|_{B(\mathscr{H})} \leqslant t_{i j}
$$

где матрица $t=\left(t_{i j}\right)_{i, j=1, \ldots, n}$ задает сжатие в пространстве $\mathbb{L}_{p}\left(m_{1}, \ldots, m_{n} ; \mathbb{R}\right)$, т. е.

$$
\sum_{j=1}^{n} m_{j}\left|\sum_{i=1}^{n} t_{i j} x_{i}\right|^{p} \leqslant \sum_{i=1}^{n} m_{i}\left|x_{i}\right|^{p} \quad \text { при }\left(x_{1}, \ldots, x_{n}\right) \in \mathbb{R}^{n} .
$$

Сжатие $T=\left(T_{i j}\right)$ в пространстве $\mathbb{L}_{p}\left(m_{1}, \ldots, m_{n} ; \mathscr{H}\right)$ называется симметричным, если все операторы $T_{i j}$ являются самосопряженными.

Пусть $(Z, \mathscr{F}, \mu)$ - пространство с мерой и $G=\left(E_{1}, \ldots, E_{n}\right)$ - разбиение пространства $Z$, причем $\mu\left(E_{j}\right)=m_{j}, j=1, \ldots, n$. Очевидно, что пространство $\mathbb{L}_{p}\left(m_{1}, \ldots, m_{n} ; \mathscr{H}\right)$ изоморфно пространству $\mathbb{L}_{p}(Z, S, \mu ; \mathscr{H})$, где $S$ - алгебра подмножеств пространства $Z$, порожденная разбиением $G$ (причем изометрический изоморфизм задается формулой $\left.\left(\xi_{1}, \ldots, \xi_{n}\right) \rightarrow \sum_{j=1}^{n} \xi_{j} 1_{E_{j}}\right)$.

Оператор условного ожидания $\mathscr{E}^{G}: \mathbb{L}_{p}(Z, \mathscr{F}, \mu ; \mathscr{H}) \rightarrow \mathbb{L}_{p}(Z, S, \mu ; \mathscr{H})$ задается формулой

$$
\left(\mathscr{E}^{G} f\right)(x)=\frac{1}{\mu\left(E_{j}\right)} \int_{E_{j}} f(z) \mu(d z) \quad \text { при } x \in E_{j} .
$$

Пусть в пространстве $\mathbb{L}_{p}(Z, \mathscr{F}, \mu ; \mathscr{H})$ задано сжатие $T$ и $\mathscr{E}=\mathscr{E} G-$ введенный выше оператор. Рассмотрим $\mathscr{E} T$ как оператор, действующий только в $\mathbb{L}_{p}(Z, S, \mu ; \mathscr{H}) \sim \mathbb{L}_{p}\left(m_{1}, \ldots, m_{n} ; \mathscr{H}\right)$. Таким образом, для подходящей матрицы $\left(T_{i j}\right)$ мы можем записать

$$
((\mathscr{E} T) f)_{j}=\sum_{i=1}^{n} T_{i j} f_{i}, \quad j=1, \ldots, n,
$$

где $f=\left(f_{1}, \ldots, f_{n}\right), f_{j} \in \mathscr{H}$.

Введя эти обозначения, мы можем сформулировать наш основной результат.

Теорема. Пусть $T$ - сжатие в пространстве $\mathbb{L}_{p}(Z, \mathscr{F}, \mu ; \mathscr{H}), 1<p<\infty$, $p \neq 2$. Предположим, что выполнено следующее условие: 
для любого конечного разбиения $G=\left(E_{1}, \ldots, E_{n}\right)$ пространства $Z\left(\right.$ с $\left.\mu\left(E_{j}\right)=m_{j}\right)$ и соответствующего условного ожидания $\mathscr{E} G$ сжатие $\mathscr{E}^{G}$ т в пространстве $\mathbb{L}_{p}(Z, S, \mu, \mathscr{H}) \sim \mathbb{L}_{p}\left(m_{1}, \ldots, m_{n}, \mathscr{H}\right)$ симметрично и мажорируется сжатием.

Тогда чезаровские средние

$$
\frac{1}{n} \sum_{k=0}^{n-1}\left(T^{k} f\right)(z), \quad n=1,2, \ldots,
$$

сильно сходятся в $\mathscr{H}$ почти везде относительно меры $\mu$.

НАБРОСОК ДОКАЗАТЕЛЬСТвА. Доказательство по существу следует идеям Акоглу [1]. Основной шаг состоит в построении обратимой изометрии $Q$, которая является дилатацией для сжатия в пространстве $\mathbb{L}_{p}\left(m_{1}, \ldots, m_{n} ; \mathscr{H}\right)$, на некотором большем пространстве $\mathbb{L}_{p}(Z, \mathscr{F}, \mu ; \mathscr{H})$ при помощи условных ожиданий. С помощью этого процесса Акоглу сводит доказательство к одному результату Ионеску-Тульчи [6] (см. также улучшенные версии в работах де ла Торре [5] и Кана [4]). Мы действуем аналогичным образом и сводим доказательство к результатам Камберна [3], Сурура [7] и Ассани [2]. А именно, как показано в [2, Corollary I.16] (см. также [3] и [7]), для обратимой изометрии $Q$ в пространстве $\mathbb{L}_{p}(Z, \mathscr{F}, \mu ; \mathscr{H})$, где $1<p<\infty, p \neq 2$, имеет место следующее неравенство:

$$
\left(\int_{Z} \sup _{n}\left\|\frac{1}{n} \sum_{k=0}^{n-1}\left(Q^{k} f\right)(z)\right\|_{\mathscr{H}}^{p} \mu(d z)\right)^{1 / p} \leqslant \frac{p}{p-1}\left(\int_{Z}\|f(z)\|_{\mathscr{H}}^{p} \mu(d z)\right)^{1 / p} .
$$

Таким образом, нашу теорему можно вывести из теоремы II.6 работы [2] при помощи подходящего аппроксимационного рассуждения.

Переходя к описанию некоторых деталей доказательства, зафиксируем положительные числа $m_{1}, \ldots, m_{n}$ и предположим, что $1<p<\infty, p \neq 2$. Пусть $T=\left(T_{i j}\right)_{i, j=1, \ldots, n}-$ симметричное сжатие в пространстве $\mathbb{L}_{p}\left(m_{1}, \ldots, m_{n} ; \mathscr{H}\right)$, которое мажорируется сжатием: $\left\|T_{i j}\right\| \leqslant t_{i j}$, где $t=\left(t_{i j}\right)$ - сжатие в пространстве $\mathbb{L}_{p}\left(m_{1}, \ldots, m_{n} ; \mathbb{R}\right) ;$ предположим на время, что сжатие $t=\left(t_{i j}\right)$ удовлетворяет условиям

$$
t_{i j}>0 \text { при всех } i, j \text { и }\|t\|_{p}=1 \text { (ср. [1]). }
$$

Тогда $\left(t_{i j}\right)^{-1} T_{i j}-$ сжатие в $\mathscr{H}$. Известно, что любое самосопряженное сжатие $K$ в гильбертовом пространстве имеет вид $K=\frac{1}{2}\left(U+U^{*}\right)$, где $U-$ унитарный оператор. Достаточно положить $U=K+i\left(\mathbf{1}-K^{2}\right)^{1 / 2}$. Таким образом, мы можем записать

$$
T_{i j}=\frac{1}{2} t_{i j}\left(U_{i j}^{(1)}+U_{i j}^{(2)}\right), \quad i, j=1, \ldots, n,
$$

где $U_{i j}^{(\alpha)}$ - унитарные операторы в $\mathscr{H}(i, j=1, \ldots, n ; \alpha=1,2)$.

В следующих нескольких строках мы ссылаемся на часть конструкции Акоглу [1], сохраняя его обозначения. Выберем множество $Z$ и преобразование $\tau: Z \rightarrow Z$ в точности так, как описано в работе [1, p. 1077]. Теперь разобьем $J_{i j}$ (где $J_{i j}$ определено в [1]) на две равные части $J_{i j}^{(\alpha)}, \alpha=1,2$, и положим 
$R_{i j}^{(\alpha)}=I_{i} \times J_{i j}^{(\alpha)}$, так что $\left|R_{i j}^{(\alpha)}\right|=\frac{1}{2}\left|R_{i j}\right|=\frac{1}{2} \eta_{i j}(\alpha=1,2)$. При $z \in Z, f \in \mathbb{L}_{p}$ положим

$$
(Q f)(z)=[\rho(z)]^{1 / p} V\left(\tau^{-1} z\right)\left[f\left(\tau^{-1} z\right)\right]
$$

где $V(z)=U_{i j}^{(\alpha)}$ на множестве $R_{i j}^{(\alpha)}$, а $U_{i j}^{(\alpha)}$ - унитарные операторы из формулы (9). Очевидно, что $Q$ - обратимая изометрия в $\mathbb{L}_{p}(Z, \mathscr{F}, \mu ; \mathscr{H})$.

Пусть $f-$ функция, заданная на $Z$ и зависящая только от $x$-координаты, т. е. $f(z)=F(x)$ при $z=(x, y)$.

Проведя необходимые вычисления, получаем

$$
\begin{aligned}
\frac{1}{\mu\left(E_{j}\right)} \int_{E_{j}}(Q f)(z) \mu(d z) & =\sum_{i=1}^{n} \frac{1}{2} t_{i j}\left[U_{i j}^{(1)}+U_{i j}^{(2)}\right] \frac{1}{\mu\left(E_{i}\right)} \int_{E_{i}} f(z) \mu(d z) \\
& =\sum_{i=1}^{n} T_{i j}\left(\frac{1}{\mu\left(E_{i}\right)} \int_{E_{i}} f(z) \mu(d z)\right), \quad j=1, \ldots, n .
\end{aligned}
$$

Отождествляя пространство $\mathbb{L}_{p}\left(m_{1}, \ldots, m_{n} ; \mathscr{H}\right)$ с $\mathbb{L}_{p}(Z, S, \mu ; \mathscr{H})$, где $S-$ алгебра, порожденная разбиением $\left(E_{1}, \ldots, E_{n}\right)$, и обозначая через $\mathscr{E}$ условное ожидание, соответствующее этому разбиению, можно переписать (11) в виде

$$
\mathscr{E} Q f=T \mathscr{E} f, \quad f \in \mathbb{L}_{p}(Z, \mathscr{F}, \mu ; \mathscr{H}) .
$$

Таким образом, для функции $f \in \mathbb{L}_{p}\left(m_{1}, \ldots, m_{n} ; \mathscr{H}\right) \sim \mathbb{L}_{p}(Z, S, \mu ; \mathscr{H})$ имеем

$$
\mathscr{E} Q^{k} f=T^{k} f, \quad k=0,1, \ldots
$$

(при дополнительном предположении (8)). Поскольку для изометрии $Q$ справедливо неравенство (7), мы получаем

$$
\left(\int_{Z} \sup _{n}\left\|\frac{1}{n} \sum_{k=0}^{n-1}\left(T^{k} f\right)(z)\right\|_{\mathscr{H}}^{p} \mu(d z)\right)^{1 / p} \leqslant \frac{p}{p-1}\left(\int_{Z}\|f(z)\|_{\mathscr{H}}^{p} \mu(d z)\right)^{1 / p} .
$$

Теперь нетрудно избавиться от предположения (8), ср. [1].

Чтобы доказать нашу теорему в полной общности, возьмем сжатие $T$ в пространстве $\mathbb{L}_{p}(Z, \mathscr{F}, \mu ; \mathscr{H})$, удовлетворяющее условию (5).

Предположим, что для $T$ неравенство $(7)$ не выполнено. Тогда существует положительное целое число $N$ и функция $f \in \mathbb{L}_{p}(Z, \mathscr{F}, \mu ; \mathscr{H})$, такие, что

$$
\int_{Z} \max _{1 \leqslant n \leqslant N}\left\|\frac{1}{n} \sum_{k=0}^{n-1}\left(T^{k} f\right)(z)\right\|_{\mathscr{H}}^{p} \mu(d z)>\left(\frac{p}{p-1}\right)^{p} \int_{Z}\|f(z)\|_{\mathscr{H}}^{p} \mu(d z) .
$$

Для произвольного $\delta>0$ найдем условное ожидание $\mathscr{E}$, соответствующее некоторому конечному разбиению $\left(E_{1}, \ldots, E_{n}\right)$ пространства $Z$, такое, что

$$
\int_{Z}\left\|f_{k}(z)-g_{k}(z)\right\|_{\mathscr{H}}^{p} \mu(d z)<\delta, \quad k=1, \ldots, N
$$

где

$$
g_{k}=\frac{1}{k} \sum_{\nu=0}^{k-1}(\mathscr{E} T)^{\nu} \mathscr{E} f, \quad f_{k}=\frac{1}{k} \sum_{\nu=0}^{k-1} T^{\nu} f \quad \text { (cp. [1, Lemma 3.1]). }
$$

Тогда

$$
\int_{Z}\left|\left\|f_{k}(z)\right\|_{\mathscr{H}}-\left\|g_{k}(z)\right\|_{\mathscr{H}}\right|^{p} \mu(d z)<\delta, \quad k=1, \ldots, N
$$


По непрерывности нормы в $\mathbb{L}_{p}(Z, \mu)$ для любого $\eta>0$ найдется такое $\delta>0$, что

$$
\int_{Z}\left|\left\|f_{k}(z)\right\|_{\mathscr{H}}^{p}-\left\|g_{k}(z)\right\|_{\mathscr{H}}^{p}\right| \mu(d z)<\eta .
$$

Для любого $\epsilon>0$ мы можем выбрать такое $\eta>0$, что

$$
\int_{Z}\left|\max _{1 \leqslant k \leqslant N}\left\|f_{k}(z)\right\|_{\mathscr{H}}^{p}-\max _{1 \leqslant k \leqslant N}\left\|g_{k}(z)\right\|_{\mathscr{H}}^{p}\right| \mu(d z)<\epsilon .
$$

Учитывая определения функций $f_{k}$ и $g_{k}$, из $(15),(16)$ и стандартных соображений непрерывности получаем

$$
\int_{Z} \max _{1 \leqslant n \leqslant N}\left\|\frac{1}{n} \sum_{k=0}^{n-1}(\mathscr{E} T)^{k} \mathscr{E} f\right\|_{\mathscr{H}}^{p} \mu(d z)>\left(\frac{p}{p-1}\right)^{p} \int_{Z}\|f(z)\|_{\mathscr{H}}^{p} \mu(d z),
$$

что противоречит предположению (5). Теорема доказана.

\section{ЛиТЕРАТУРА}

1. Akcoglu M. A. Canad. J. Math., 27, 1075-1082 (1975). 2. Assani I. Ann. Inst. Henri Poincaré, 23, 209-224 (1987). 3. Cambern M. Pacific J. Math., 55, 9-17 (1974). 4. Charn-Huen Kan. Canad. J. Math., 30, 1206-1214 (1978). 5. de la Torre A. Canad. J. Math., 28, 1073-1075 (1976). 6. Ionescu-Tulcea A. Bull. Amer. Math. Soc., 70, 366-371 (1964). 7. Sourour A. R. J. Funct. Anal., 30, 276-285 (1978).

Faculty of Mathematics, University of Łódź, Poland e-mail: rjajte@math.uni.lodz.pl

Поступило в редакцию 24 декабря 2004 г. 